\title{
Identification of Hostile Hemodynamics and Geometries of Cerebral Aneurysms: A Case-Control Study
}

\author{
(D).J. Chung, (DF. Mut, (DC.M. Putman, (D) F. Hamzei-Sichani, (DW. Brinjikji, (DD. Kallmes, (D) C.M. Jimenez, and (D).R. Cebral
} O-

\begin{abstract}
BACKGROUND AND PURPOSE: Hostile hemodynamic conditions and geometries are thought to predispose aneurysms for instability and rupture. This study compares stable, unstable, and ruptured aneurysms while controlling for location and patient characteristics.
\end{abstract}

MATERIALS AND METHODS: The hemodynamics and geometries of 165 stable, 65 unstable, and 554 ruptured aneurysms were compared. Hemodynamics was modeled using image-based computational fluid dynamics. Case-control pairs were selected matching aneurysm location, patient age, and sex. Paired Wilcoxon tests were used to compare hemodynamic and geometric variables among different aneurysm groups. The pairing was repeated 100 times, and the combined $P$ values were calculated and adjusted for multiple testing.

RESULTS: Ruptured aneurysms had lower minimum wall shear stress $(P=.03)$, higher maximum wall shear stress $(P=.03)$, more concentrated $(P=.03)$ and mean oscillatory shear stress $(P=.03)$, higher maximum velocity $(P=.03)$, and more complex flows (vortex core-line length, $P=.03)$ than stable aneurysms. Similarly, unstable aneurysms had more concentrated shear stress $(P=.04)$ and more complex flows (vortex core-line length, $P=.04$ ) than stable aneurysms. Compared with stable aneurysms, ruptured aneurysms were larger (size ratio, aneurysm size/vessel size, $P=.03$ ), more elongated (aspect ratio, $P=.03$ ), and irregular (nonsphericity index, $P=.03$ ). Similarly, unstable aneurysms were larger (size ratio, $P=.04$ ), more elongated (aspect ratio, $P=.04$ ), and irregular (bulge location, $P=.04$; area-weighted Gaussian curvature; $P=.04$ ) than stable aneurysms. No significant differences were found between unstable and ruptured aneurysms.

CONCLUSIONS: Unstable and ruptured aneurysms have more complex flows with concentrated wall shear stress and are larger, more elongated, and irregular than stable aneurysms, independent of aneurysm location and patient sex and age.

ABBREVIATIONS: $\mathrm{AR}=$ aspect ratio; Asize = aneurysm maximum size; $\mathrm{BL}=$ bulge location; $\mathrm{CORELEN}=$ vortex core-line length, flow complexity; $\mathrm{CP}=$ conicity parameter; GAA = area-weighted Gaussian curvature; LSA = percentage area under low WSS; $\max =$ maximum; min = minimum; NSI = nonsphericity index; OSI = oscillatory shear stress; $\mathrm{SCI}=$ concentrated shear stress; SizeR = size ratio (aneurysm size/vessel size); Vmax = maximum velocity; VOR = volume-to-ostium ratio; WSS $=$ wall shear stress

M any factors appear to be related to aneurysm rupture risk: location, size, morphology, hemodynamics, perianeurysmal environment, and patient history, among others. ${ }^{1}$ It is wellunderstood that aneurysm rupture-risk estimation is based on the combination of such factors and is a complex process. ${ }^{2}$ The American Heart Association and American Stroke Association guide-

Received October 16, 2017; accepted after revision June 27, 2018.

From the Bioengineering and Mechanical Engineering Departments (B.J.C., F.M., J.R.C.), Volgenau School of Engineering George Mason University, Fairfax, Virginia; Interventional Neuroradiology Unit (C.M.P.), Inova Fairfax Hospital, Falls Church,

Virginia; Department of Neurological Surgery (F.H.-S.), University of Massachusetts, Worcester, Massachusetts; Department of Radiology (W.B., D.K.), Mayo Clinic, Rochester, Minnesota; and Neurosurgery Department (C.M.J.), University of Antioquia, Medellin, Colombia.

This work was partially supported by the National Institutes of Health-National Institute of Neurological Disorders and Stroke grant No. R21NS094780.

Please address correspondence to Juan R. Cebral, PhD, Bioengineering Department, Volgenau School of Engineering, George Mason University, 4400 University Dr, MSN 2A1, Fairfax, VA 22030; e-mail: jcebral@gmu.edu

lines for intracranial aneurysm management recommend that in addition to the size and location of the aneurysm and patient's age and health status, it may be reasonable to consider the morphologic and hemodynamic characteristics of the aneurysm when discussing its rupture risk. ${ }^{3}$ Thus, identification of "hostile" hemodynamic conditions and geometries that predispose aneurysms to instability and rupture is important to discriminate high- and low-risk aneurysms and guide the management strategy for each individual patient. ${ }^{4}$ Additionally, understanding adverse conditions and their connections to the underlying mechanisms of aneurysm progression, instability, and rupture is valuable for designing new therapeutic strategies targeting those mechanisms. ${ }^{5}$

Several previous studies have analyzed possible associations between hemodynamics and rupture ${ }^{6-9}$ and between aneurysm

\footnotetext{
-- Indicates open access to non-subscribers at www.ajnr.org

Indicates article with supplemental on-line tables.

http://dx.doi.org/10.3174/ajnr.A5764
} 
morphology and rupture. ${ }^{1,10}$ One of the most important limitations of most cross-sectional studies comparing ruptured and unruptured aneurysms is the inability to discriminate stable and unstable unruptured aneurysms. ${ }^{8,9}$ On the other hand, the most important limitation of most longitudinal studies is the small sample size drawn from a highly selected population. ${ }^{11-13}$ Additionally, only a few studies control for aneurysm location and/or patient characteristics. ${ }^{14}$ These limitations may cause confusion and apparent conflicts among the results of different studies. ${ }^{15}$

The goal of this study was to compare the hemodynamics and geometries of stable, unstable, and ruptured aneurysms while controlling for location and patient characteristics.

\section{MATERIALS AND METHODS \\ Data}

Our data base contains $>2000$ cerebral aneurysms imaged with $3 \mathrm{D}$ rotational angiography and basic information including aneurysm status, location, size, patient age, and sex. The main contributors have been Inova Fairfax Hospital (Virginia), Mt. Sinai Medical Center (New York), and the Mayo Clinic (Minnesota). Age was divided into 3 groups: young (younger than 40 years), middle age (40-60 years), and old (older than 60 years). The images and data have been anonymized, and the study has been approved by the institutional review board of George Mason University. In the present study, a subset of 784 aneurysms was included, subdivided into the following subgroups (the distribution of aneurysms by location is presented in On-line Table 1):

1) Stable Aneurysms. Untreated unruptured aneurysms followed longitudinally without noticeable enlargement, shape change, new symptoms, or rupture for at least 1 year were considered stable. A total of 165 stable aneurysms in 85 patients were considered.

2) Unstable Aneurysms. Unruptured aneurysms were considered unstable if they either enlarged during follow-up (as determined by Sforza et $\mathrm{al}^{11}$ ) or presented with symptoms such as cranial nerve palsy indicative of aneurysm instability. A total of 65 aneurysms in 55 patients were classified as unstable, with 53 aneurysms classified as growing and 12 with instabilities related to symptoms.

3) Ruptured Aneurysms. The ruptured aneurysms group included all aneurysms confirmed as the source of subarachnoid hemorrhage. A total of 554 ruptured aneurysms in 501 patients were considered.

\section{Models}

Computational fluid dynamics models of all 2000 aneurysms in our data base have been created from the corresponding 3D rotational angiography images (at baseline for longitudinally followed aneurysms) using previously described methods. ${ }^{16}$ These models include the patient-specific vascular geometry, but because patient-specific flow conditions were not available, typical pulsatile flow conditions were derived from phase-contrast MR imaging measurements in healthy subjects and scaled with the area of the inflow vessel. ${ }^{17}$ Outflow boundary conditions consistent with flow splits given by the principle of minimal work (Murray law) were prescribed at the outlets. Vessel walls were approximated as rigid, and blood viscosity was approximated as Newtonian. The unsteady incompressible Navier-Stokes equations were numerically solved with a finite-element code developed in-house. ${ }^{18}$ All simulations were run in parallel shared-memory computers for 2 cardiac cycles. Data from the second cycle were used to quantitatively characterize the aneurysm hemodynamic environment by computing several flow variables over the aneurysm cavity, the aneurysm orifice, and the aneurysm surface. ${ }^{19}$ Additionally, several geometric variables were also computed to characterize the aneurysm morphology. ${ }^{20} \mathrm{~A}$ list of the variables considered is provided in On-line Table 2.

\section{Analysis}

Case-control studies are a common and efficient means of studying rare diseases with long latency periods, such as ruptured/ unruptured aneurysms. ${ }^{21}$ Matching of cases and controls is frequently used to control the effects of known potential confounding variables. The analysis of matched data requires specific statistical methods. The nonparametric Wilcoxon signed rank test for measured outcomes with 1:1 matching was used in the following 4 case-control studies: 1) stable-versus-ruptured aneurysms, 2) stable-versus-unstable aneurysms, 3) unstable-versus-ruptured aneurysms, and 4) unruptured-versus-ruptured aneurysms. In each study, case-control pairs were created and the mean values of hemodynamic and geometric variables of the 2 groups were statistically compared using a paired Wilcoxon test. The matching of cases and controls in each study was performed as detailed below:

1) Stable-versus-Ruptured Aneurysms. In this case, the controls (stable aneurysms) were fewer than the cases (ruptured aneurysms); therefore, the matching was performed as follows: For each stable aneurysm, a list of ruptured aneurysms with matching location, sex, and age groups was created, and one of these matching aneurysms was randomly selected. A total of 134 aneurysm pairs were thus created.

2) Stable-versus-Unstable Aneurysms. In this study, the cases (unstable aneurysms) were fewer than the controls (stable aneurysms); therefore, for each unstable aneurysm, a stable one was randomly selected from the list of stable aneurysms at the same location with matching sex and age groups. Sixty-five aneurysm pairs were created.

3) Unstable-versus-Ruptured Aneurysms. In this study, for each unstable aneurysm, a ruptured one was randomly selected from the list of ruptured aneurysms matching location, sex, and age. Sixty pairs were created.

4) Unruptured-versus-Ruptured Aneurysms. In this study, the controls (unruptured aneurysms) were fewer than the cases (ruptured aneurysms). Thus, for each unruptured aneurysm, a ruptured one was randomly selected from the list of unruptured aneurysms at the same location with matching sex and age groups. A total of 180 pairs were created.

Once the case-control pairs were created, the mean values of the hemodynamic and geometric variables of the 2 groups were statistically compared using a paired Wilcoxon test. Because the pairing processes described above involve random selection of a 
Values of hemodynamic and geometric variables in stable, unstable, and ruptured aneurysms ${ }^{\mathrm{a}}$

\begin{tabular}{|c|c|c|c|c|}
\hline \multirow[b]{3}{*}{ Variable } & \multirow[b]{3}{*}{ Stable } & \multicolumn{2}{|l|}{ Unruptured } & \multirow[b]{3}{*}{ Ruptured } \\
\hline & & \multicolumn{2}{|c|}{ Unstable } & \\
\hline & & Symptoms & Growing & \\
\hline WSSmin & $0.8 \pm 1.9$ & $0.3 \pm 0.5$ & $1.0 \pm 4.5$ & $0.4 \pm 1.3$ \\
\hline WSSmax & $223.7 \pm 187.5$ & $235.0 \pm 101.8$ & $253.7 \pm 170.2$ & $377.5 \pm 948.8$ \\
\hline WSSmean & $21.4 \pm 18.5$ & $16.6 \pm 13.8$ & $22.0 \pm 22.1$ & $23.2 \pm 28.9$ \\
\hline WSSnorm & $0.6 \pm 0.3$ & $0.4 \pm 0.3$ & $0.5 \pm 0.3$ & $0.4 \pm 0.3$ \\
\hline $\mathrm{SCl}$ & $3.78 \pm 4.76$ & $8.52 \pm 9.44$ & $5.52 \pm 4.53$ & $6.23 \pm 6.88$ \\
\hline LSA & $45.8 \pm 33.6$ & $62.9 \pm 29.7$ & $58.8 \pm 30.9$ & $51.6 \pm 33.0$ \\
\hline OSImax & $0.259 \pm 0.137$ & $0.358 \pm 0.089$ & $0.303 \pm 0.125$ & $0.317 \pm 0.114$ \\
\hline OSImean & $0.012 \pm 0.011$ & $0.014 \pm 0.008$ & $0.017 \pm 0.016$ & $0.017 \pm 0.017$ \\
\hline Q & $0.746 \pm 0.805$ & $1.360 \pm 1.368$ & $1.081 \pm 0.957$ & $0.730 \pm 0.807$ \\
\hline $\mathrm{ICl}$ & $0.716 \pm 0.763$ & $1.193 \pm 1.309$ & $1.088 \pm 0.941$ & $0.791 \pm 0.764$ \\
\hline Vmax & $62.9 \pm 31.8$ & $70.8 \pm 19.6$ & $80.4 \pm 50.0$ & $88.8 \pm 62.6$ \\
\hline VE & $9.7 \pm 6.4$ & $8.7 \pm 5.7$ & $10.1 \pm 6.1$ & $9.8 \pm 8.1$ \\
\hline SR & $226.2 \pm 176.1$ & $155.0 \pm 131.4$ & $202.9 \pm 221.2$ & $231.5 \pm 260.7$ \\
\hline VO & $307.7 \pm 241.1$ & $218.0 \pm 183.7$ & $278.9 \pm 279.0$ & $317.4 \pm 344.4$ \\
\hline CORELEN & $1.753 \pm 3.313$ & $2.726 \pm 1.827$ & $2.504 \pm 3.043$ & $2.378 \pm 2.593$ \\
\hline PODENT & $0.176 \pm 0.125$ & $0.227 \pm 0.115$ & $0.228 \pm 0.155$ & $0.207 \pm 0.137$ \\
\hline Asize & $0.687 \pm 0.524$ & $1.109 \pm 0.603$ & $0.933 \pm 0.550$ & $0.770 \pm 0.398$ \\
\hline Nsize & $0.482 \pm 0.309$ & $0.690 \pm 0.461$ & $0.577 \pm 0.311$ & $0.429 \pm 0.172$ \\
\hline SizeR & $1.834 \pm 1.395$ & $2.440 \pm 1.127$ & $2.533 \pm 1.487$ & $2.492 \pm 1.373$ \\
\hline$A R$ & $0.879 \pm 0.607$ & $1.308 \pm 0.828$ & $1.200 \pm 0.831$ & $1.304 \pm 0.691$ \\
\hline VOR & $0.894 \pm 3.355$ & $1.220 \pm 1.310$ & $1.460 \pm 2.906$ & $0.919 \pm 1.559$ \\
\hline $\mathrm{El}$ & $0.273 \pm 0.036$ & $0.268 \pm 0.021$ & $0.263 \pm 0.026$ & $0.273 \pm 0.025$ \\
\hline NSI & $0.202 \pm 0.051$ & $0.229 \pm 0.080$ & $0.211 \pm 0.041$ & $0.246 \pm 0.054$ \\
\hline $\mathrm{BF}$ & $1.167 \pm 0.465$ & $1.414 \pm 0.478$ & $1.377 \pm 0.574$ & $1.322 \pm 0.441$ \\
\hline$B L$ & $0.290 \pm 0.161$ & $0.431 \pm 0.170$ & $0.361 \pm 0.144$ & $0.355 \pm 0.163$ \\
\hline $\mathrm{CR}$ & $0.762 \pm 0.144$ & $0.793 \pm 0.083$ & $0.816 \pm 0.118$ & $0.785 \pm 0.100$ \\
\hline $\mathrm{CP}$ & $0.210 \pm 0.161$ & $0.069 \pm 0.170$ & $0.139 \pm 0.144$ & $0.145 \pm 0.163$ \\
\hline UI & $0.238 \pm 0.144$ & $0.207 \pm 0.083$ & $0.184 \pm 0.118$ & $0.215 \pm 0.100$ \\
\hline GAA & $16.339 \pm 17.905$ & $5.861 \pm 4.444$ & $7.893 \pm 8.034$ & $12.763 \pm 13.717$ \\
\hline
\end{tabular}

Note:-Q indicates aneurysm flow rate; ICl, inflow concentration index; VE, mean aneurysm velocity; SR, mean aneurysm shear rate; VO, mean aneurysm velocity; PODENT, POD entropy, flow instability; POD, proper orthogonal decomposition; Nsize, neck maximum size; El, ellipticity index; BF, bottleneck factor; CR, convexity ratio; UI, undulation index; WSSnorm, normalized wall shear stress.

${ }^{a}$ Values are given as mean \pm SD.

case-control pair from the list of available matches, a bootstrapping or random sampling with a replacement approach was used $^{22}$ to improve the robustness of the analysis. Specifically, the pairing and tests were repeated 100 times, and the $P$ values were combined with the Wilkinson method. ${ }^{23}$ A difference was then considered statistically significant if the combined $P$ value was $<.05$. The combined $P$ values were then adjusted for multiple testing using the false discovery rate method. ${ }^{24}$ It was verified that 100 pairings were enough by performing 200 pairings and verifying that the significance of the comparisons did not change. All statistical analyses were performed by using R scripts ( $\mathrm{R}$ statistical and computing software; http://www.r-project.org).

\section{RESULTS}

The general hemodynamic and geometric characteristics of the aneurysm groups considered in this study are summarized in the Table. This Table lists the mean values and SDs of each variable over each group.

\section{Stable-versus-Ruptured Aneurysms}

The statistically significant differences $(P<.05$ before correcting for multiple testing) between stable and ruptured aneurysms are summarized in Fig 1. The bars represent the ratio of the mean values of the ruptured aneurysm group over the mean values of the stable aneurysm group, and the error bars represent the vari- ability of these ratios over the 100 random pairings. The mean and SDs of all variables over the 2 groups, along with the corresponding $P$ values, are listed in On-line Table 3.

These results indicate that compared with stable aneurysms, after adjustment for multiple testing, ruptured aneurysms tend to have hemodynamic environments characterized by lower minimum wall shear stress (WSSmin, $P=.03$ ), higher maximum WSS (WSSmax, $P=.03$ ), more concentrated shear stress distributions (SCI, $P=.03$ ), more mean oscillatory shear stress (OSImean, $P=.03$ ), higher maximum velocity (Vmax, $P=.03$ ), and more complex flow patterns (vortex core-line length [CORELEN], $P=.03$ ). Additionally, ruptured aneurysms had geometries characterized by larger size (size ratio, aneurysm size/vessel size [SizeR], $P=.03$ ), more elongated shapes (aspect ratio $[\mathrm{AR}], P=.03$ ), and more irregular shapes (nonsphericity index [NSI], $P=.03$ ).

\section{Stable-versus-Unstable Unruptured Aneurysms}

Comparisons of hemodynamic and geometric variables between stable and unstable unruptured aneurysms are presented in Online Table 4 and are summarized in Fig 2 . These results indicate that unstable aneurysms tend to have more SCI $(P=.04)$ and more complex flow patterns (CORELEN, $P=.04$ ) than stable aneurysms. Unstable aneurysms also tend to have larger areas 


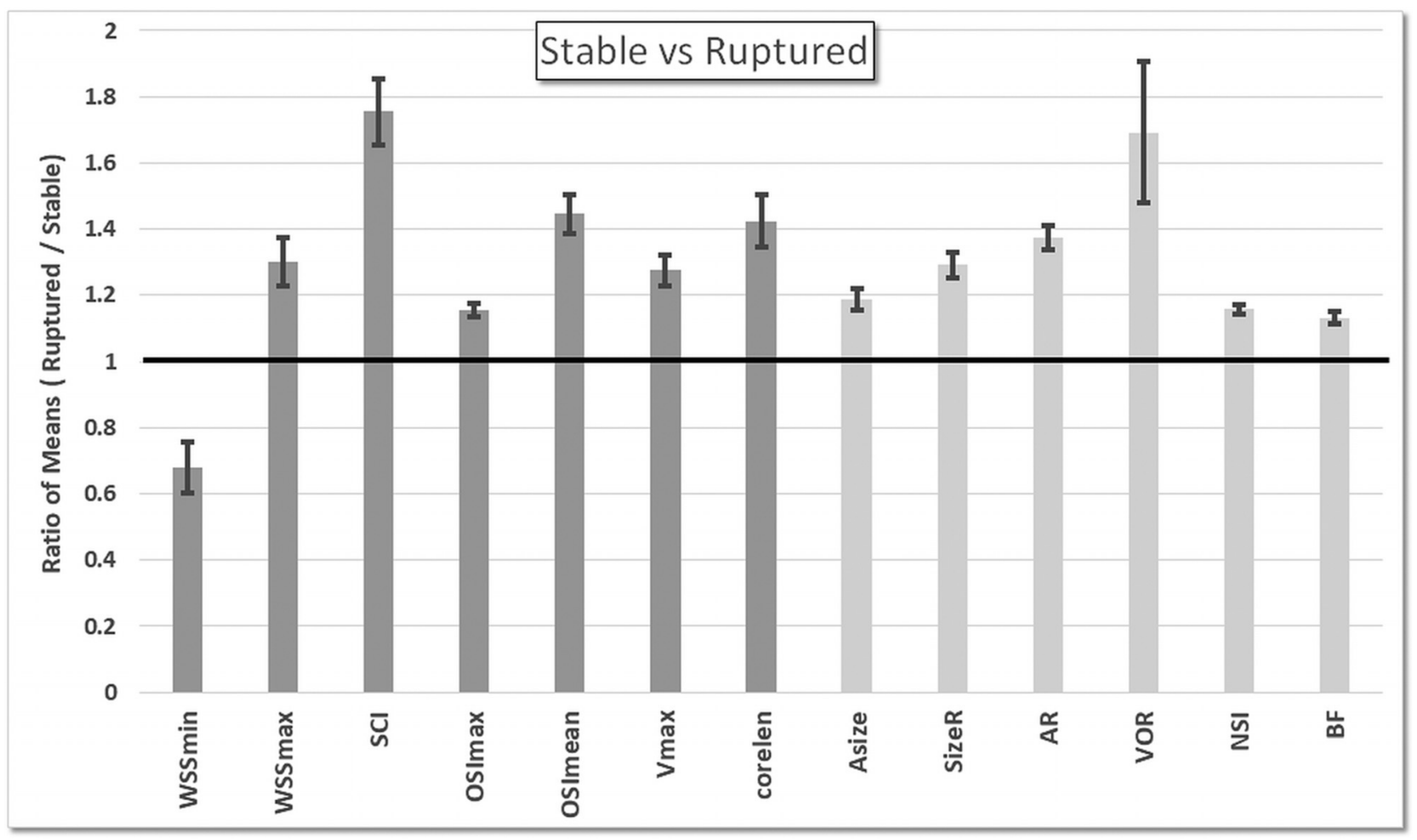

FIG 1. Ratios of the mean values of the ruptured aneurysm group over mean values of the stable aneurysm group. Only hemodynamic (dark gray) and geometric (light gray) variables that were significantly different between these groups $(P<.05$ before adjusting for multiple testing) are included.

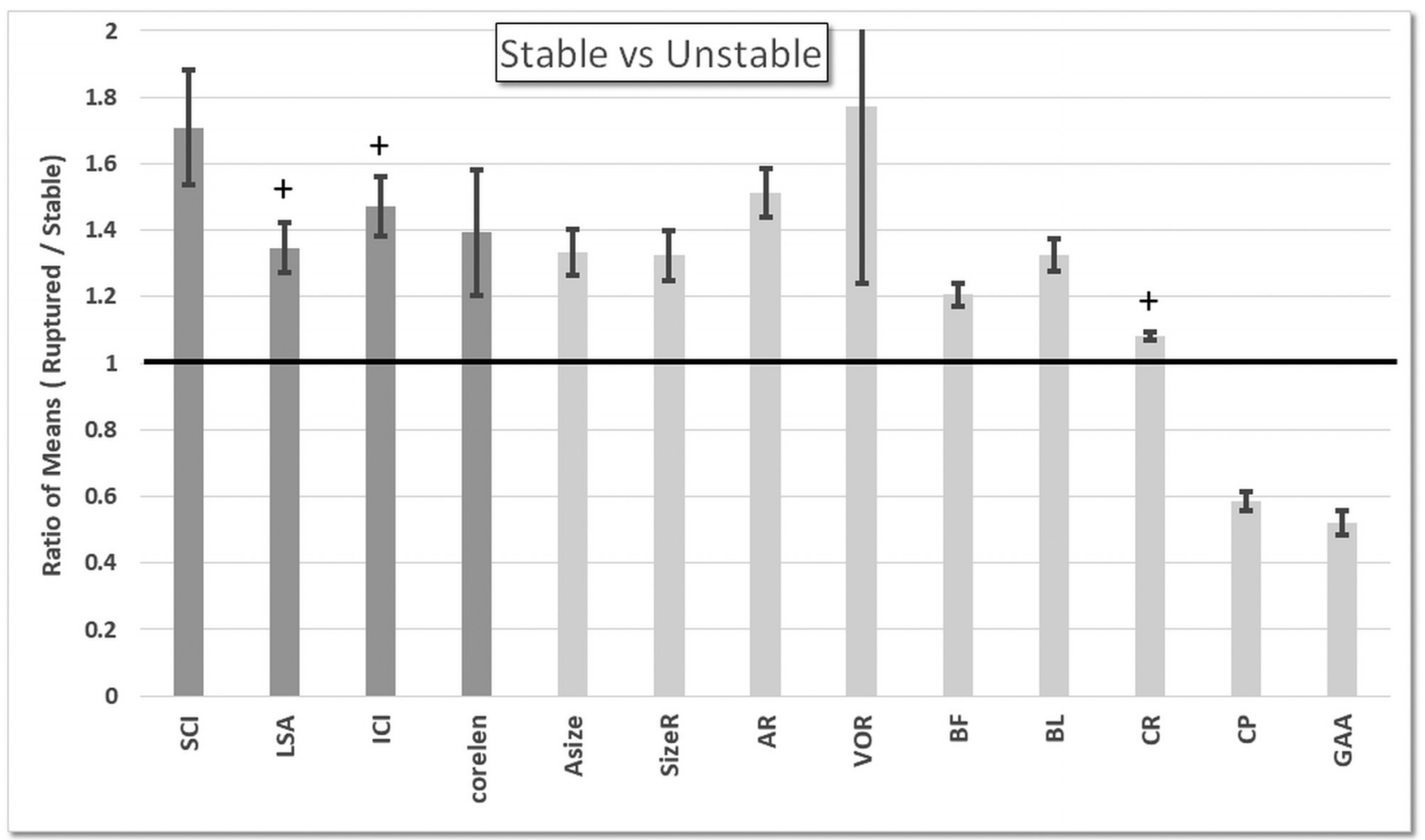

FIG 2. Ratios of mean values of the unstable aneurysm group over mean values of the stable aneurysm group. Only hemodynamic (dark gray) and geometric (light gray) variables that were significantly different $(P<.05$ before adjusting for multiple testing) or marginally significant $(P<.1$ before adjusting for multiple testing; marked with a plus sign) are included. ICl indicates inflow concentration index; BF, bottleneck factor; CR, convexity ratio.

under low WSS (percentage area under low WSS [LSA], $P=.10$ before adjustment) and more concentrated inflow jets (inflow concentration index, $P=.07$ before adjustment), but these asso- ciations did not reach statistical significance. Geometrically, unstable aneurysms are larger (aneurysm maximum size [Asize], $P=.04$; SizeR, $P=.04$ ), more elongated (AR, $P=.04$; volume- 


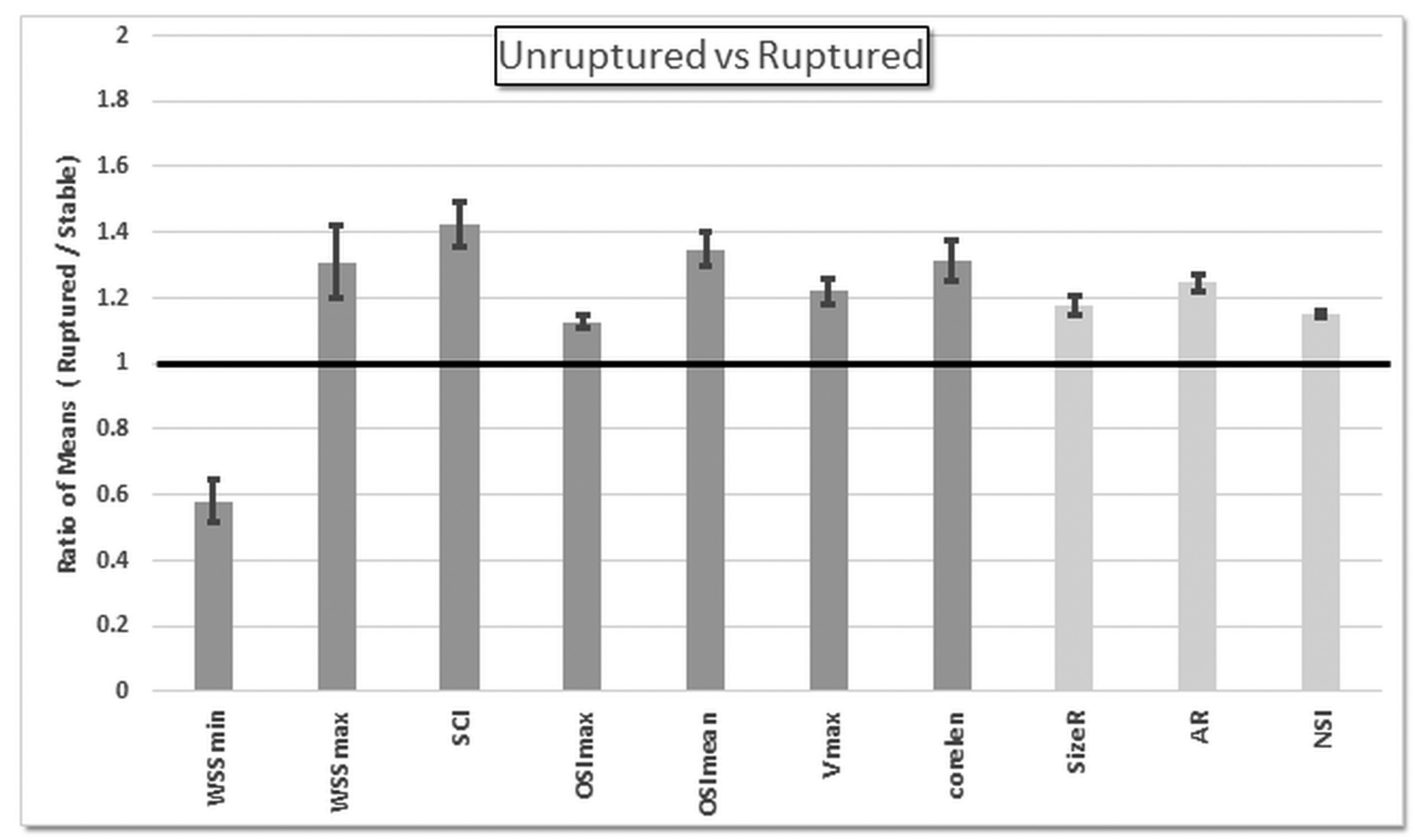

FIG 3. Ratios of mean values of the ruptured aneurysm group over mean values of the unruptured aneurysm group. Only hemodynamic (dark gray) and geometric (light gray) variables that were significantly different between these groups $(P<.05$ before adjusting for multiple testing) are included.

to-ostium ratio [VOR], $P=.04)$, and more irregular in shape (bulge location $[\mathrm{BL}], P=.04$; conicity parameter $[\mathrm{CP}], P=.04$; area-weighted Gaussian curvature; $[\mathrm{GAA}], P=.04)$ than stable aneurysms.

\section{Unstable-versus-Ruptured Aneurysms}

No significant differences between unstable unruptured aneurysms and matched ruptured aneurysms were found, including both hemodynamic and geometric variables (see the results in On-line Table 5).

\section{Unruptured-versus-Ruptured Aneurysms}

Results of the comparison of all unruptured aneurysms included in this study (stable and unstable combined) against matching ruptured aneurysms are presented in On-line Table 6 and summarized in Fig 3. Again, ruptured aneurysms had more SCI $(P=$ .04 ) and more complex flow patterns (CORELEN, $P=.04$ ) with higher $\operatorname{Vmax}(P=.04)$ compared with unruptured aneurysms. They also tended to have lower WSSmin $(P=.02$ before adjustment) and larger WSSmax ( $P=.02$ before adjustment), as well as more maximum oscillatory shear stress (OSImax, $P=.02$; OSImean, $P=.02$ before adjustment), but these associations became only marginally significant $(P=.06)$ after adjusting for multiple testing. Geometrically, ruptured aneurysms were more elongated (AR, $P=.04$ ) and more irregular (NSI, $P=.04$ ) and tended to be larger (SizeR, $P=.02$ before adjustment), but the latter association became marginally significant $(P=.06)$ after adjusting for multiple testing.

\section{DISCUSSION}

The current study attempts to characterize hostile aneurysm hemodynamics and geometry that could predispose aneurysms to instability and rupture while controlling for location, sex, and age. This knowledge is important for improved understanding of the mechanisms of wall degeneration and aneurysm progression.

The differences observed between stable and ruptured aneurysm pairs allow us to identify hemodynamic and geometric characteristics that are more prevalent in ruptured aneurysms. Our results indicate that these hostile hemodynamic characteristics include extreme values of wall shear stress (low and high), concentrated and oscillatory shear stress distributions, and complex intrasaccular flow patterns. Adverse aneurysm geometries include large, elongated, and irregular shapes. These characteristics are illustrated with 3 examples presented in Fig 4. Additionally, a simple correlation analysis was performed to identify variables correlated to one another. Groups of correlated variables (whose pair-wise regression coefficient was greater than 0.80) are presented in On-line Tables 7-9. Most interesting, no correlations were found between hemodynamic and geometric variables in this sample.

Differences between stable and unstable aneurysms were less pronounced but in the same general direction as those between stable and ruptured aneurysms. Specifically, unstable aneurysms had more complex flow patterns with concentrated wall shear stress than stable aneurysms, and they were larger and more elongated and irregular. These features suggest that unstable and ruptured aneurysms differ from stable aneurysms in similar ways. 

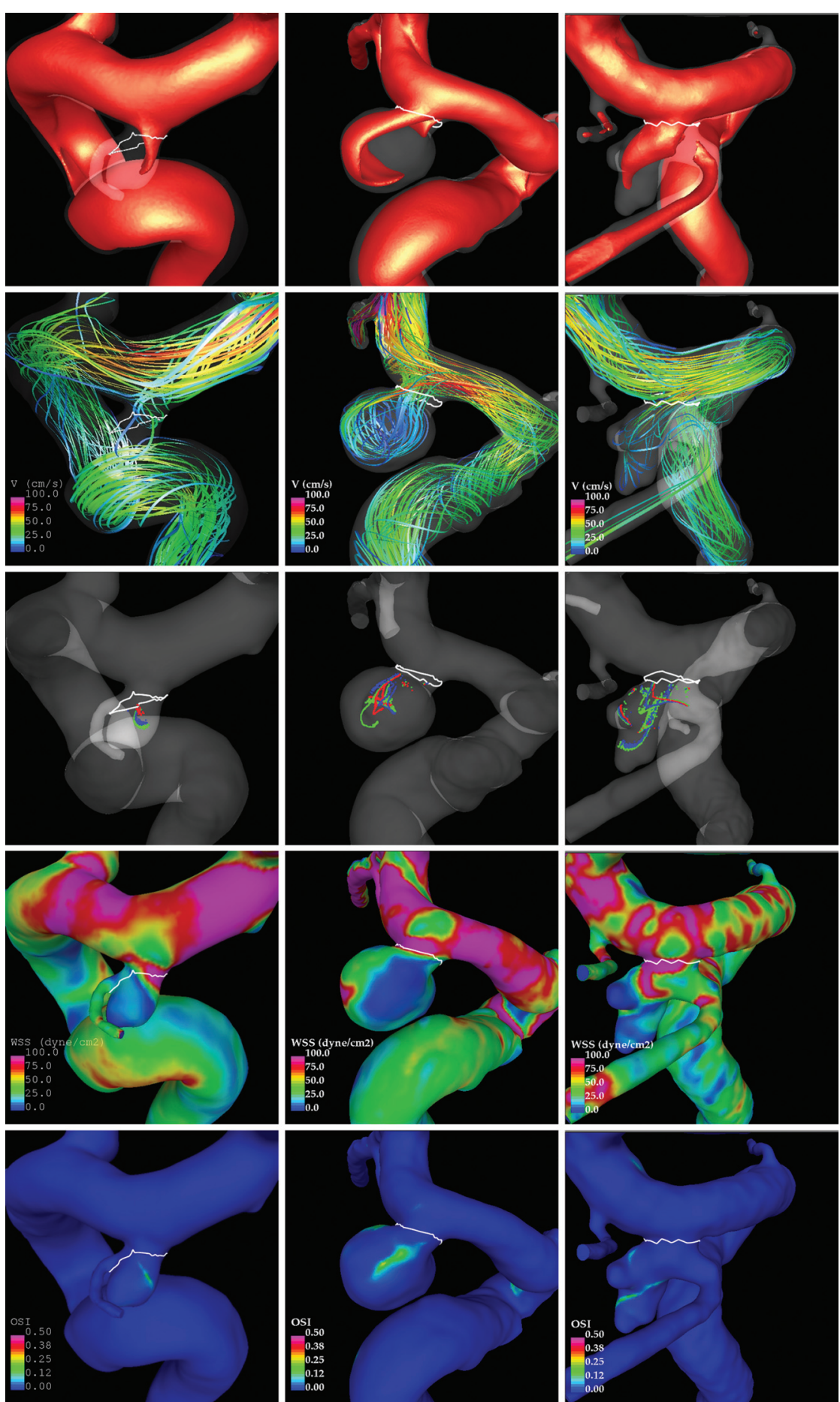

FIG 4. Examples of stable (left), unstable (middle), and ruptured (right) aneurysms at a single location (posterior communicating artery). Visualizations show, from upper to lower: inflow jet, flow pattern, vortex core lines at 4 instants during the cardiac cycle, wall shear stress, and oscillatory shear index.

Furthermore, no significant differences were found between unstable and ruptured aneurysms. These observations are consistent with a study ${ }^{25}$ that found differences between symptomatic and asymptomatic posterior communicating artery aneurysms that were similar to the differences between symptomatic and ruptured aneurysms at this location. This finding suggests that hemodynamic environments and shapes of unstable aneurysms may resemble those of ruptured aneurysms, but further studies with larger samples are needed to confirm this conjecture. Finally, when comparing all unruptured aneurysms (stable and unstable combined) against ruptured aneurysms, differences similar to those observed between stable and ruptured aneurysms were observed. This is likely due to the larger proportion of stable aneurysms in the unruptured group.

Several studies have compared stable and unstable unruptured aneurysms, but without controlling for location or patient characteristics. One such study did not find significant geometric or hemodynamic differences (149 stable, 20 unstable), ${ }^{26}$ while 2 other studies found differences in geometric (70 stable, 23 unstable) ${ }^{27}$ and hemodynamic (17 stable, 16 unstable) characteristics. ${ }^{11}$ Our findings agree with these latter studies. Two other studies found larger LSAs in unstable aneurysms compared with stable aneurysms after matching location and size (12 stable, 12 unstable) ${ }^{14}$ as well as sex and age (12 stable, 12 unstable). ${ }^{28}$ In general, our results show similar trends, but LSA did not reach statistical significance.

In summary, the results of our study suggest that hostile hemodynamic environments (ie, more prevalent in ruptured and unstable aneurysms than in stable aneurysms) are characterized by complex flows and concentrated wall shear stress distributions, while adverse geometries include larger and more elongated and irregular shapes. This finding leads us to propose that through geometric and flow analysis, there are ways to a priori identify unruptured aneurysms that will evolve toward instability and will therefore be at a higher risk of rupture. Additionally, our study suggests that lumping stable and unstable aneurysms into a single group of unruptured aneurysms (as in most cross-sectional studies) still allows characterization of adverse conditions likely because of the higher prevalence of stable aneurysms in the unruptured population.

Our study has several limitations. Computational fluid dynamics models are based on several assumptions and approximations. ${ }^{19}$ Our sample size and available data were not large enough to allow us to match and control for more patient characteristics or to obtain more statistical significance. The unstable group contained growing and cerebral palsy cases, which should be studied separately with larger samples. This study identified several trends that suggest that hostile hemodynamics and geometries could 
help identify aneurysms at higher risk of instability and rupture and should be considered in studies aiming at further understanding the underlying mechanisms that contribute to the evolution of a cerebral aneurysm from stable to unstable and ultimately ruptured status.

\section{CONCLUSIONS}

Unstable and ruptured aneurysms have more complex flows with concentrated wall shear stress and are larger, more elongated, and irregular than stable aneurysms, independent of aneurysm location, sex, and age. These adverse conditions could be used to identify unruptured aneurysms at higher risk of rupture and should be taken into account in studies of the mechanisms responsible for aneurysm wall degradation and progression to instability and rupture.

Disclosures: Fernando Mut-RELATED: Grant: National Institutes of Health, Comments: research grant. ${ }^{*}$ Waleed Brinjikji-UNRELATED: Consultancy: Johnson and Johnson; Stock/Stock Options: Marblehead Medical LLC; Travel/Accommodations/Meeting Expenses Unrelated to Activities Listed: Johnson and Johnson. Juan R. Cebral—RELATED: Grant: National Institutes of Health *; UNRELATED: Grants/ Grants Pending: National Institutes of Health, Philips Healthcare.* *Money paid to the Institution.

\section{REFERENCES}

1. Chien A, Sayre J, Viñuela F. Comparative morphological analysis of the geometry of ruptured and unruptured aneurysms. Neurosurgery 2011;69:349-56 CrossRef Medline

2. Greving JP, Wermer MJ, Brown RD Jr, et al. Development of the PHASES score for prediction of risk of rupture of intracranial aneurysms: a pooled analysis of six prospective cohort studies. Lancet Neurol 2014;13:59-66 CrossRef Medline

3. Connolly ES Jr, Rabinstein AA, Carhuapoma JR, et al; American Heart Association Stroke Council, Council on Cardiovascular Radiology and Intervention, Council on Cardiovascular Nursing, Council on Cardiovascular Surgery and Anesthesia, Council on Clinical Cardiology. Guidelines for the management of aneurysmal subarachnoid hemorrhage: a guideline for healthcare professionals from the American Heart Association/American Stroke Association. Stroke 2012;43:1711-37 CrossRef Medline

4. Cebral J, Ollikainen E, Chung BJ, et al. Flow conditions in the intracranial aneurysm lumen are associated with inflammation and degenerative changes of the aneurysm wall. AJNR Am J Neuroradiol 2017;38:119-26 CrossRef Medline

5. Frösen J, Tulamo R, Paetau A, et al. Saccular intracranial aneurysm: pathology and mechanisms. Acta Neuropathol 2012;123:773-86 CrossRef Medline

6. Cebral JR, Castro MA, Burgess JE, et al. Characterization of cerebral aneurysms for assessing risk of rupture by using patient-specific computational hemodynamics models. AJNR Am J Neuroradiol 2005;26:2550-59 Medline

7. Shojima M, Oshima M, Takagi K, et al. Magnitude and role of wall shear stress on cerebral aneurysm: computational fluid dynamic study of $\mathbf{2 0}$ middle cerebral artery aneurysms. Stroke 2004;35: 2500-05 CrossRef Medline

8. Xiang J, Natarajan SK, Tremmel M, et al. Hemodynamic-morphologic discriminants for intracranial aneurysm rupture. Stroke 2011; 42:144-52 CrossRef Medline

9. Cebral JR, Mut F, Weir J, et al. Quantitative characterization of the hemodynamic environment in ruptured and unruptured brain aneurysms. AJNR Am J Neuroradiol 2011;32:145-51 CrossRef Medline

10. Dhar S, Tremmel M, Mocco J, et al. Morphology parameters for intracranial aneurysm rupture risk assessment. Neurosurgery 2008; 63:185-96; discussion 196-97 CrossRef Medline

11. Sforza DM, Kono K, Tateshima S, et al. Hemodynamics in growing and stable cerebral aneurysms. J Neurointerv Surg 2016;8:407-12 CrossRef Medline

12. Tateshima $\mathrm{S}$, Tanishita $\mathrm{K}, \mathrm{O}$ Omura $\mathrm{H}$, et al. Intra-aneurysmal hemodynamics during the growth of unruptured aneurysm: in vitro study using longitudinal CT angiogram database. AJNR Am J Neuroradiol 2007;28:622-27 Medline

13. Boussel L, Rayz V, McCulloch C, et al. Aneurysm growth occurs at region of low wall shear stress: patient-specific correlation of hemodynamics and growth in a longitudinal study. Stroke 2008;39: 2997-3002 CrossRef Medline

14. Brinjikji W, Chung BJ, Jimenez C, et al. Hemodynamic differences between unstable and stable unruptured aneurysms independent of size and location: a pilot study. J Neurointerv Surg 2017;9:376-80 CrossRef Medline

15. Kallmes DF. Point: CFD—computational fluid dynamics or confounding factor dissemination. AJNR Am J Neuroradiol 2012;33: 396-96 CrossRef Medline

16. Cebral JR, Castro MA, Appanaboyina S, et al. Efficient pipeline for image-based patient-specific analysis of cerebral aneurysm hemodynamics: technique and sensitivity. IEEE Trans Med Imaging 2005;24:457-67 CrossRef Medline

17. Cebral JR, Castro MA, Putman CM, et al. Flow-area relationship in internal carotid and vertebral arteries. Physiol Meas 2008;29:585-94 CrossRef Medline

18. Mut F, Aubry R, Löhner R, et al. Fast numerical solutions of patientspecific blood flows in 3D arterial systems. Int J Num Meth Biomed Eng 2010;26:73-85 CrossRef Medline

19. Mut F, Löhner R, Chien A, et al. Computational hemodynamics framework for the analysis of cerebral aneurysms. Int J Num Meth Biomed Eng 2011;27:822-39 CrossRef Medline

20. Raghavan ML, Ma B, Harbaugh RE. Quantified aneurysm shape and rupture risk. J Neurosurg 2005;102:355-62 CrossRef Medline

21. Niven DJ, Berthiaume LR, Fick GH, et al. Matched case-control studies: a review of reported statistical methodology. Clin Epidemiol 2012;4:99-110 CrossRef Medline

22. Efron B, Tibshirani RJ. An Introduction to the Bootstrap. Boca Raton: Chapman and Hall; 1993

23. Wilkinson B. A statistical consideration in psychological research. Psychol Bull 1951;48:156-58 CrossRef Medline

24. Benjamini Y, Hochberg Y. Controlling the false discovery rate: a practical and powerful approach to multiple testing. J R Stat Soc Series B 1995;57:289-300

25. Lv N, Yu Y, Xu J, et al. Hemodynamic and morphological characteristics of unruptured posterior communicating artery aneurysms with oculomotor nerve palsy. J Neurosurg 2016;125: 264-68 CrossRef Medline

26. Ramachandran M, Retarekar R, Raghavan ML, et al. Assessment of image-derived risk factors for natural course of unruptured cerebral aneurysms. J Neurosurg 2016;124:288-95 CrossRef Medline

27. Chien $\mathrm{A}, \mathrm{Xu} \mathrm{M}$, Yokota $\mathrm{H}$, et al. Nonsphericity index and size ratio identify morphologic differences between growing and stable aneurysms in a longitudinal study of $\mathbf{9 3}$ cases. AJNR Am J Neuroradiol 2018 Jan 25. [Epub ahead of print] CrossRef Medline

28. Skodvin $T \varnothing$, Evju $\varnothing$, Helland CA, et al. Rupture prediction of intracranial aneurysms: a nationwide matched case-control study of hemodynamics at the time of diagnosis. J Neurosurg 2017 Nov 3:1-7. [Epub ahead of print] CrossRef Medline 